\title{
The Role of p53 Expression in Patients with RAS/BRAF Wild-Type Metastatic Colorectal Cancer Receiving Irinotecan and Cetuximab as Later Line Treatment
}

\author{
Pina Ziranu ${ }^{1}$ - Eleonora Lai ${ }^{1}$. Marta Schirripa ${ }^{2}$ - Marco Puzzoni ${ }^{1} \cdot$ Mara Persano $^{1}$ - Andrea Pretta ${ }^{1,3,4} \cdot$ Giada Munari $^{2}$. \\ Nicole Liscia $^{1,3} \cdot$ Valeria Pusceddu $^{1} \cdot$ Fotios Loupakis ${ }^{2} \cdot$ Laura Demurtas $^{1} \cdot$ Michela Libertini $^{5} \cdot$ Stefano Mariani $^{1}$. \\ Marco Migliari ${ }^{1} \cdot$ Marco Dubois $^{1} \cdot$ Riccardo Giampieri $^{6} \cdot$ Giovanni Sotgiu $^{7} \cdot$ Angelo Paolo Dei Tos $^{8} \cdot$ Sara Lonardi $^{2}$. \\ Alberto Zaniboni ${ }^{5} \cdot$ Matteo Fassan $^{8} \cdot$ Mario Scartozzi $^{1}$
}

Accepted: 20 April 2021 / Published online: 10 May 2021

(c) The Author(s) 2021

\begin{abstract}
Background Preclinical and clinical data indicate that p53 expression might modulate the activity of the epidermal growth factor receptor (EGFR), influencing response/resistance to anti-EGFR monoclonal antibodies. However, the association between p53 status and clinical outcome has not been clarified yet.

Objective In our study, we evaluated the role of p53 expression in patients with $R A S / B R A F$ wild-type metastatic colorectal cancer (mCRC) receiving irinotecan/cetuximab in an exploratory and a validation cohort.

Patients and Methods p53 expression was analysed in patients with $R A S / B R A F$ wild-type mCRC receiving second-line or third-line irinotecan/cetuximab. Survival distribution was assessed by the Kaplan-Meier method, while the log-rank test was used for survival comparison.

Results Among 120 patients with RAS/BRAF wild-type mCRC included in our analysis, 52 (59\%) and 19 (59\%) patients showed p53 overexpression in the exploratory and validation cohort, respectively. In the exploratory cohort, low p53 expression was correlated with better median progression-free survival (hazard ratio $0.39 ; p<0.0001$ ), median overall survival (hazard ratio: $0.23 ; p<0.0001)$ and response rate $(p<0.0001)$. These results were confirmed by data of the validation cohort where we observed better median progression-free survival (hazard ratio: $0.48 ; p=0.0399$ ), median overall survival (hazard ratio: $0.26 ; p=0.0027)$ and response rate $(p=0.0007)$ in patients with p53 normal expression $\mathrm{mCRC}$.

Conclusions In our study, p53 overexpression was associated with anti-EGFR treatment resistance in patients with $R A S /$ $B R A F$ WT mCRC, as confirmed in a validation cohort. Larger studies are needed to validate the role of p53 and investigate EGFR cross-talk in these patients.
\end{abstract}

\section{Introduction}

The transmembrane tyrosine kinase epidermal growth factor receptor (EGFR or HER1, ErB1) belongs to the ErbB family along with ErbB-2, ErbB-3 and ErbB-4a and is a key driver of cell proliferation, survival, adhesion, migration and differentiation [1-3]. Deregulation of the EGFR pathway was observed in several malignancies such as colorectal, non-small cell lung, breast, ovary, renal, head and neck,

Pina Ziranu and Eleonora Lai contributed equally to this work.

Mario Scartozzi

marioscartozzi@gmail.com

Extended author information available on the last page of the article pancreatic, prostate, cervical and bladder cancer $[1,4,5]$. In this setting, EGFR overexpression/upregulation and hyperactivation might be responsible for the promotion of tumour cell growth, resistance to apoptosis, synthesis of angiogenic and growth factors and metastatic spread. As a consequence, EGFR blockade plays an established role as an anti-cancer strategy, especially in colorectal cancer (CRC), non-small cell lung cancer, and head and neck tumours [6-14]. Presently, the anti-EGFR monoclonal antibodies cetuximab and panitumumab represent the cornerstone of treatment for $R A S$ wild-type (WT) metastatic CRC (mCRC) [15, 16].

Unfortunately, not all patients with RAS WT mCRC are sensitive to EGFR-targeting agents and resistance to these drugs is still an open issue [17-21]. Several potential mechanisms for the lack of efficacy of anti-EGFR have been 


\section{Key Points}

TP53 status may affect the response to anti-epidermal growth factor receptor (EGFR) therapies as a consequence of p53 activity on the EGFR promoter, and TP53 mutation is often associated with its overexpression.

Our analysis suggests a predictive/prognostic role of abnormal p53 expression in patients with metastatic colorectal cancer treated with anti-EGFR therapy.

The finding of p53 overexpression as a resistance mechanism to anti-EGFR therapies represents a further step toward personalised treatment.

investigated beyond $N-R A S$ and $K-R A S$ mutational status, such as BRAF V600E mutation [22-26], PI3KCA exon 20 mutation, PTEN loss [22, 27-29], Stat3 and Akt phosphorylation [22, 30-32], HER2 amplification [22, 33-36], HER3 expression [37], IGF1R activation [22, 38, 39], MET amplification $[22,40]$, altered vascular endothelial growth factor/ vascular endothelial growth factor receptor expression [22, 41], EGFR gene copy number alteration and EGFR methylation $[42,43]$, but for most of these no definitive data are available.

Among the potential mechanisms of resistance, data suggest that TP53 mutations might play a role in tumour cell sensitivity to anti-EGFR antibodies. In fact, recent studies have suggested that activation of the EGFR pathway leads to malignant transformation only if the $\mathrm{p} 53$ protein is inactivated [44].

The $\mathrm{p} 53$ protein, which is encoded by the tumour suppressor gene p53 (TP53) located in chromosome 17p, is one of the most important tumour suppressors. Acting as a zinccontaining transcription factor [45-49], it regulates downstream genes involved in DNA repair, cell-cycle arrest and apoptosis [50-54]. More specifically, in the event of cellular stress signals such as genotoxic damage, oncogene activation, hypoxia or loss of intercellular adhesion, p53 takes on an active tetrameric form and stops cell-cycle progression and induces DNA repair, senescence and cell death through apoptosis to preserve the genomic integrity. Consequently, p53 has a crucial role in the regulation of cell proliferation and cancer development inhibition; for its pivotal role in protection from tumour development, it has been defined as "the guardian of the genome" [54-61].

Mutations or deletions of the TP53 gene can be found in nearly $50 \%$ of human cancers and they lead to an impaired tumour suppressor function [62]. Tumours harbouring a TP53 mutation are usually characterised by genomic instability and poor prognosis [54, 63, 64]. Moreover, it was shown that in p53 mutants, the loss of WT p53 function is also associated with the gain of new oncogenic roles promoting cancer, metastasis, inhibition of apoptosis and drug resistance [64-67].

Mutations in TP53 usually occur in $40-60 \%$ of patients with CRC. There are well-known logistical difficulties and resource limitations associated with direct sequencing of the TP53 gene. Therefore, most studies have used immunohistochemistry to detect mutant p53, with the assumption that a mutation is often associated with its overexpression. However, the lack of expression is generally indicative of WT TP53. Only in limited cases, a complete loss of p53 expression is associated with truncating mutations and loss of heterozygosity ( $\mathrm{LOH})$. Several trials documented conflicting results regarding the association between p53 overexpression and clinical outcome [68-71].

The predictive function of TP53 mutations in patients with mCRC treated with targeted therapies has not been established so far. However, some evidence suggests that TP53 status may affect the response to anti-EGFR therapies as a consequence of the activity of p53 on the promoter of the EGFR [72-77]. However, findings from previous analyses investigating the role of p53 in this setting were contradictory. Based on these considerations, we designed our study with the aim to assess the predictive/ prognostic role of p53 abnormal expression in patients with mCRC treated with anti-EGFR therapy by using a validation cohort.

\section{Methods}

\subsection{Patients and Methods}

Patients with $R A S$ and $B R A F$ WT mCRC treated with second-line or third-line irinotecan and cetuximab were included in our retrospective analysis. Between May 2011 and May 2018, subjects treated at the University of Cagliari were included in the exploratory cohort, while subjects treated at Istituto Oncologico Veneto constituted the validation cohort. Tumour samples were retrospectively tested for p53 immunohistochemical (IHC) expression with the aim of evaluating the correlation with clinical outcome in terms of overall survival (OS), progressionfree survival (PFS), response rate (RR) and disease control rate (DCR).

The analysis of p53 expression were performed on formalin-fixed and paraffin-embedded tumour samples for all patients using an anti-p53 antibody (DO7, prediluted; Leica Biosystems, Newcastle upon Tyne, UK) and the Bond Polymer Refine Detection Kit (Leica Biosystems) on a BOND-MAX automated IHC stainer (Leica Biosystems), as instructed by the manufacturer. The cut-off 
point for p53 expression was set as $30 \%$, as previously described. In detail, if $30 \%$ or more of the malignant nuclei were positive, the tumour was scored as positive, while if fewer than $30 \%$ of the nuclei were stained, the tumour was scored as negative (Fig. 1) [78-80]. For study purposes, right-sided and left-sided colorectal primary tumours were defined as proximal or distal to the splenic flexure.

\subsection{Statistical Analysis}

Statistical analysis was performed with the MedCalc Statistical Software Version 14.10.2 (MedCalc Software bvba, Ostend, Belgium; http://www.medcalc.org; 2014). The association between categorical variables was estimated by the Fisher exact test for categorical binomial variables or by the chi-square test in all other instances. Survival probability over time was estimated by the Kaplan-Meier method. Significant differences in the probability of survival between the strata were evaluated by the log-rank test. The independent role of variables that were statistically significant at a univariate analysis was assessed with a logistic regression analysis.

Overall survival was defined as the time interval between the date of the beginning of cetuximab/irinotecan treatment to death or the last follow-up visit for patients who were lost at follow-up. Progression-free survival was defined as the interval between the date of the beginning of cetuximab/ irinotecan treatment to death, first sign of clinical progression or the last follow-up visit for patients who were lost at follow-up.
Response rate was defined as the percentage of patients who achieved a partial or complete response to treatment according to RECIST Version 1.1. Disease control rate was defined as the percentage of patients with stable disease or partial/complete response to treatment.

Based on the results from the 88 patients of the exploratory cohort, we tried to validate the findings in a validation cohort. Then, we identified the validation group sample size according to good/poor prognosis group ratio and survival analysis, from the exploratory cohort. To validate the difference in terms of a 12-month rate among poor prognosis patients (around 15\%) and good prognosis patients (around $70 \%$ ), as we obtained in the exploratory cohort (assuming a probability alpha of 0.05 and a beta of 0.10 ), with a two group ratio of 0.7 , the required sample size would have been 32 patients $(13+19)$, using a "comparison of proportion test".

\section{Results}

\subsection{Patient Characteristics}

Globally, 120 patients with RAS/BRAF WT mCRC were included in our analysis, 88 in the exploratory cohort, treated at the University of Cagliari and 32 in the validation cohort, treated at Istituto Oncologico Veneto, between May 2011 and May 2018. Patient baseline characteristics are detailed in Tables 1 and 2.
Fig. 1 Representative p53 immunohistochemical staining in RAS/BRAF wild-type colorectal adenocarcinomas. Diffuse overexpression in a primary colorectal carcinoma (a) and in a hepatic metastatic tumour (b); note the absence of p53 immunostaining in the surrounding normal colon mucosa and hepatic parenchyma. c A p53 overexpressing mucinous colorectal adenocarcinoma. d, e Heterogeneous and low p53 expression in two cases of primary colorectal adenocarcinoma. f A complete loss of p53 expression; note the presence of positive stromal cells that are used as positive controls of the reaction (original magnifications $\times 5, \times 10$ and $\times 20$; scale bars $100 \mu \mathrm{m})$
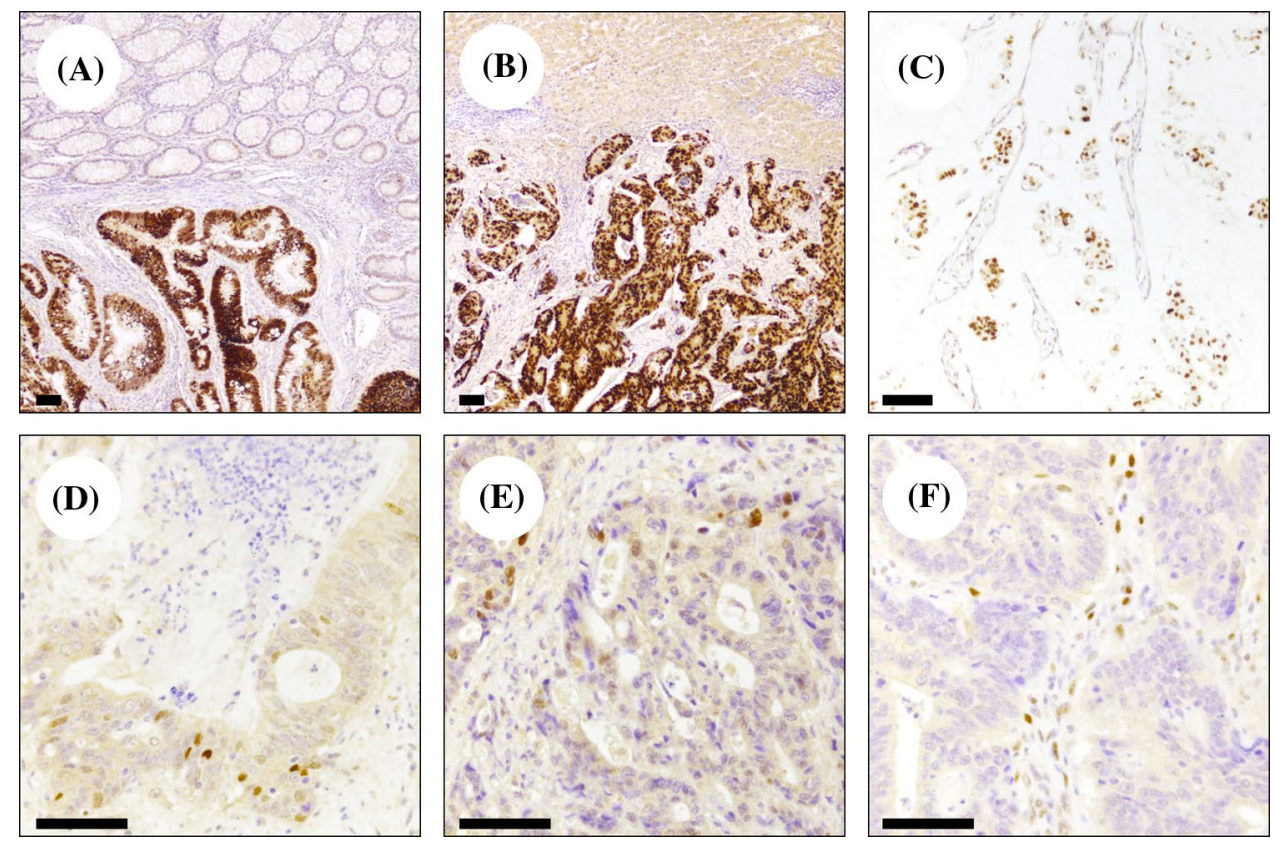
In the exploratory cohort as well as in the validation cohort, p53 IHC low expression was 41\% (36/88 and 13/32 patients, respectively). p53 IHC overexpression was found in the $59 \%$ of patients in both cohorts $(52 / 88$ patients in the exploratory cohort and 19/32 patients in the validation cohort).

In the exploratory cohort, $59 \%$ of the patients had a leftsided colorectal cancer, $46 \%$ of them had p53 low expression and 54\% p53 overexpression. Conversely, among patients with right-sided CRC, 30\% had p53 low expression and 70\% had 553 overexpression $(p=0.15)$.

The left colon site was the most common in the validation group with $62 \%$ of patients. In this subgroup of patients, $48 \%$ had p53 low expression and 52\% had p53 overexpression. Conversely, among patients with right-sided CRC, $14 \%$ had p53 low expression and 86\% had p53 overexpression ( $p$ $=0.114$ ).

\subsection{Clinical Outcomes}

At a median follow-up of 10.5 months (95\% confidence interval [CI] 9.0-13.7), in the exploratory cohort, we observed a median PFS (mPFS) of 6 months $(95 \% \mathrm{CI}$ $3.8-18.0)$ and a median OS (mOS) of 10.0 months $(95 \% \mathrm{CI}$ 9.0-13.6). Median PFS was 8.0 months (95\% CI 6.9-18.0) vs 3.0 months (95\% CI 2.9-11.1) in patients non-overexpressing p53 and in patients overexpressing p53, respectively (hazard ratio [HR]: $0.39 ; p<0.0001$ ). Median OS was 19.5 months (95\% CI $17.0-30.5)$ vs 8 months $(95 \%$ CI 5.9-9.8) in patients non-overexpressing p53 and in patients overexpressing p53, respectively; (HR: $0.23 ; p<0.0001$ ) (Fig. 2). Overall RR was 33\%. Response rate was $58 \%$ vs $15 \%$ in patients with p53 non-overexpressing tumours and in patients with $\mathrm{p} 53$ overexpressing tumours, respectively $(p<0.0001)$. Overall DCR was 59\%. Disease control rate was $86 \%$ vs $40 \%$ in patients with p53 non-overexpressing

Table 1 Patient characteristics in the exploratory and validation cohorts

\begin{tabular}{|c|c|c|c|}
\hline$N$ & $\begin{array}{l}\text { Global population } \\
120\end{array}$ & $\begin{array}{l}\text { Exploratory cohort } \\
88\end{array}$ & $\begin{array}{l}\text { Validation cohort } \\
32\end{array}$ \\
\hline Age, years, median (range) & $62(37-79)$ & $64(37-79)$ & $60(49-66)$ \\
\hline \multicolumn{4}{|l|}{ Sex, $n(\%)$} \\
\hline M & $89(74)$ & $69(78)$ & $20(62)$ \\
\hline $\mathrm{F}$ & $31(26)$ & $19(22)$ & $12(38)$ \\
\hline \multicolumn{4}{|l|}{ ECOG PS, $n(\%)$} \\
\hline $0-1$ & $105(88)$ & $76(86)$ & $29(90)$ \\
\hline$\geq 2$ & $15(12)$ & $12(14)$ & $3(10)$ \\
\hline \multicolumn{4}{|l|}{ Primary tumour location, $n(\%)$} \\
\hline Left & $72(60)$ & $52(59)$ & $20(62)$ \\
\hline Right & $48(40)$ & $36(41)$ & $12(38)$ \\
\hline \multicolumn{4}{|c|}{ Time of metastatic disease, $n(\%)$} \\
\hline Metachronous & 45 (37) & $35(39)$ & $10(32)$ \\
\hline Synchronous & $75(63)$ & $53(61)$ & $22(68)$ \\
\hline \multicolumn{4}{|c|}{ Anti-EGFR treatment line, $n(\%)$} \\
\hline II & $76(63)$ & $56(64)$ & $20(62)$ \\
\hline III & $44(37)$ & $32(34)$ & $12(38)$ \\
\hline Median OS, months & 11.8 & 10 & 15.8 \\
\hline Median PFS, months & 6 & 6 & 7 \\
\hline \multicolumn{4}{|c|}{ Best response to treatment, $n(\%)$} \\
\hline $\mathrm{CR}$ & - & - & - \\
\hline PR & $40(33)$ & $29(33)$ & $11(34$ \\
\hline SD & $39(33)$ & $23(26)$ & $16(50)$ \\
\hline PD & $41(34)$ & $36(41)$ & $5(15)$ \\
\hline \multicolumn{4}{|l|}{ p53 IHC, $n(\%)$} \\
\hline p53 over expression & $71(59)$ & $52(59)$ & $19(59)$ \\
\hline p53 low expression & $49(41)$ & $36(41)$ & $13(41)$ \\
\hline
\end{tabular}

$C R$ complete response, $E C O G$ Eastern Cooperative Oncology Group, $F$ female, $I H C$ immunohistochemistry, $M$ male, $O S$ overall survival, $P D$ progression disease, $P F S$ progression-free survival, $P R$ partial response, $S D$ stable disease 
Table 2 Patient baseline characteristics according to p53 expression

\begin{tabular}{llll}
\hline & Overall population & Left sided & Right sided \\
\hline Exploratory cohort & & & \\
Total, $n(\%)$ & 88 & $52(59 \%)$ & $36(41)$ \\
p53 overexpressed & $52(59)$ & $33(54 \%)$ & $19(70)$ \\
p53 low expression & $36(41)$ & $28(46)$ & $8(30)$ \\
Validation cohort & & & \\
Total, $n(\%)$ & 32 & $20(62)$ & $12(38)$ \\
p53 overexpressed & $19(59)$ & $13(52)$ & $6(86 \%)$ \\
p53 low expression & $13(41)$ & $12(48)$ & $1(14)$ \\
\hline
\end{tabular}

tumours and in patients with p53 overexpressing tumours, respectively $(p<0.0001)$ (Table 3$)$.

These results were confirmed in the validation cohort, where we observed an mPFS of 7 months (95\% CI 4.6-12.0) and an mOS of 15.8 months (95\% CI 9.5-28.0). Response rate was $34 \%$, and DCR was $84 \%$. Response rate was $69 \%$ in patients with low p53 and $11 \%$ in patients with abnormal p53 ( $p=0.0007)$; DCR was $100 \%$ vs $74 \%$ for patients with low p53 and abnormal p53 tumours, respectively $(p=0.0475)$ (Table 3). Median OS was 18 months in patients with p53 low expression (95\% CI 13.6-28.0) vs 11.46 months in patients with p53 overexpression (95\% CI 8.2-18.0; HR: $0.26 ; p=0.0027)$ and mPFS was 8.1 months in patients with low p53 (95\% CI 6.5-12.0) vs 5.8 months in patients with abnormal p53 (95\% CI 3.0-11.4; HR: 0.48; $p=0.0399$ ) (Fig. 3).
Regarding the tumour sidedness, patients with left-sided CRC had better outcomes than patients with left-sided CRC in the exploratory cohort, with an mOS of 12.5 months (95\% CI 9.9-19.0) vs 8 months (95\% CI 5.9-9.8), respectively (HR: $0.5 ; p=0.0232$ ). Finally, while not statistically significant, patients with left-sided CRC had better outcomes than patients with right-sided CRC in the validation cohort with 16.0 months (95\% CI 13.5-28) vs 8.9 months (95\% CI 3.4-9.0), respectively (HR: 0.3; $p=0.067$ ) (Fig. 4).

Therefore, $\mathrm{p} 53$ expression and sidedness showed a statistically significant correlation with OS at univariate analysis in both cohorts. At the multivariate analysis, in the exploratory cohort, p53 expression (Exp (b) 4.5750, $p<0.0001)$ and primary tumour location (Exp (b) 2.0820, $p=0.0078$ ) maintained an independent role. In the validation cohort, only p53 expression $(\operatorname{Exp}(b) 5.0367, p=0.0050)$ maintained an independent role.

Furthermore, to validate the prognostic role and the predictive effect of p53 in patients with $R A S / B R A F$ WT mCRC treated with anti-EGFR, we analysed the survival data from the first-line palliative therapy in both cohorts. At a median follow-up of 25.1 months (95\% CI 20.5-28.5), in the exploratory cohort, a significant difference in mOS was found between patients non-overexpressing p53 and patients overexpressing p53 [30.2 months (95\% CI 26.9-47.9) vs 17.1 (95\% CI 13.8-44.9), $p<0.0001$, respectively]. These results were confirmed in the validation cohort with an mOS of 34.7 months (95\% CI $25.8-40)$ vs 22.5 (95\% CI $17-34), p=0.0003$ (Fig. 5). Finally, a non-statistically significant trend towards better mPFS was found in patients
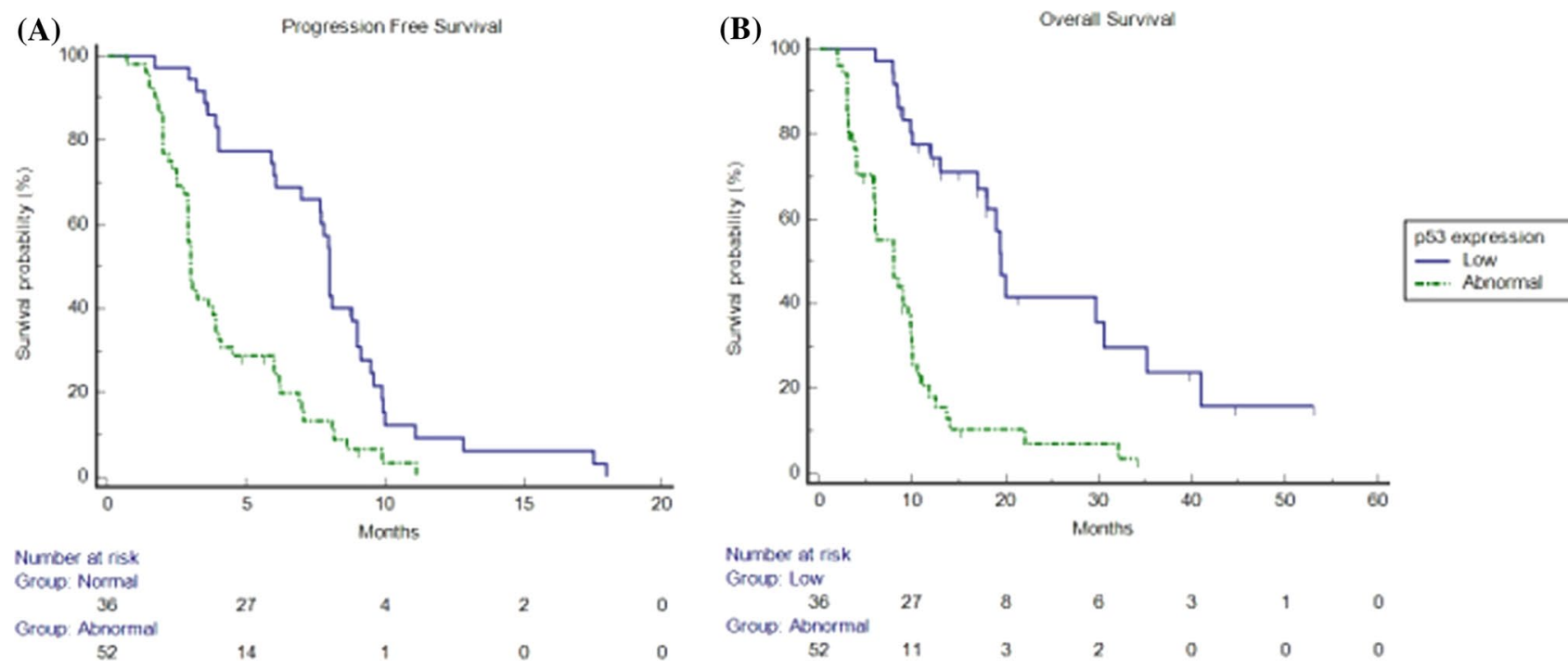

Number at risk Grow: Low

$\begin{array}{ccccccc}36 & 27 & 8 & 6 & 3 & 1 & 0 \\ \text { Group. Atonormal } & & & & & & \\ 52 & 11 & 3 & 2 & 0 & 0 & 0\end{array}$

Fig. 2 Kaplan-Meier progression-free survival and overall survival in patients overexpressing p53 (dotted line) and in patients non-overexpressing p53 (continuous line) in the exploratory cohort. a Median progression-free survival was 8.0 months $(95 \%$ confidence interval [CI] 6.9-18.0) vs 3.0 months (95\% CI 2.9-11.1) in patients overex- pressing p53 and in patients non-overexpressing p53, respectively (hazard ratio $0.39 ; p<0.0001$ ). b Median overall survival was 19.5 months (95\% CI $17.0-30.5)$ vs 8 months (95\% CI 5.9-9.8) in patients overexpressing p53 and in patients non-overexpressing p53, respectively (hazard ratio: $0.23 ; p<0.0001$ ) 
Table 3 Response in patients of the exploratory and validation cohorts in overall population, in non-overexpressing p-53 tumours and in overexpressing p53 tumours

\begin{tabular}{llll}
\hline & Overall population & $\begin{array}{l}\text { Overexpressing } \\
\mathrm{p} 53\end{array}$ & $\begin{array}{l}\text { Non-over- } \\
\text { expressing } \\
\mathrm{p} 53\end{array}$ \\
\hline \multicolumn{2}{l}{ Exploratory cohort, $n(\%)$} & & $21(58)$ \\
ORR & $29(33)$ & $8(15)$ & $31(86)$ \\
DCR & $52(59)$ & $21(40)$ & $9(69)$ \\
Validation cohort, $n(\%)$ & & $13(100)$ \\
ORR & $11(34)$ & $2(11)$ & $14(74)$ \\
DCR & $27(84)$ & 140
\end{tabular}

$D C R$ disease control rate, $O R R$ overall response rate

non-overexpressing p53 vs patients overexpressing p53 in the exploratory cohort [11 months (95\% CI 10.6-20.9) vs 8 months (95\% CI 7.9-20), $p=0.135$ ) respectively] and the validation cohort [13.1 months (95\% CI 11.8-20.4) vs 11.7 months (95\% CI 8-18.9), $p=0.1933$ ), respectively] (data not shown).

\section{Discussion and Conclusions}

The emergence of drug resistance represents the major limitation to the development and use of molecularly targeted cancer therapies. In this scenario, only a few studies tried to identify the prognostic and the predictive role of p53 in patients treated with anti-EGFR therapies.
Preclinical data suggest that both WT and mutant TP53 can modulate EGFR expression in various tumours. LudesMeyers et al. showed that WT and mutant TP53 use different mechanisms to activate EGFR transcription. They identified the binding site for WT TP53 in an EGFR promoter genespecific sequence and observed that the p53 DNA-binding capacity seemed to be related to this gene transcription activation; furthermore, this sequence appeared to be the same target site of positive and negative regulators of EGFR promoter activity. Conversely, mutant TP53 did not require the WT p53-binding site for EGFR promoter transactivation but the oligomerisation domain after recruitment by other transcription factors. As mutant p53 is often overexpressed in tumour cells, this could lead to strong and persistent activation of growth factor genes, such as high EGFR levels and consequent aberrant cell proliferation [72-76]. A further study demonstrated that the loss of p53 in normal human keratinocytes is responsible for increased EGFR expression by a mechanism involving YY1 and Sp1 and does not require p53 binding to the EGFR promoter [77]. In summary, preclinical findings show that p53 modulates EGFR promoter activity both directly by DNA binding and indirectly through other transcription factors.

Other clinical studies evaluated the co-expression of mutant p53 and EGFR mutations/overexpression in different tumours, suggesting a correlation with poor prognosis and a lack of response to tyrosine kinase inhibitors, chemotherapy and radiotherapy in non-small cell lung cancer, head and neck, and hepatocellular malignancies [81-84].
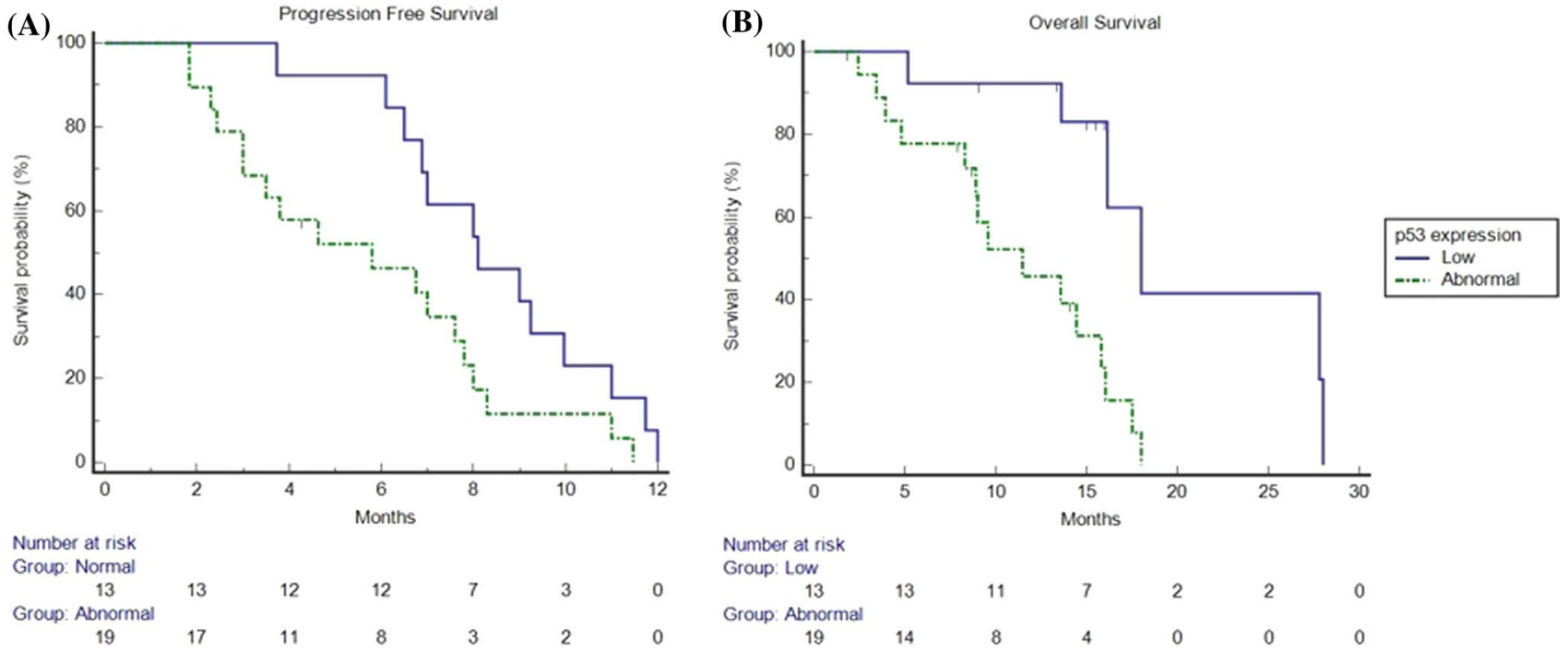

Fig. 3 Kaplan-Meier progression-free survival and overall survival in patients overexpressing p53 (dotted line) and in patients non-overexpressing p53 (continuous line) in the validation cohort. a Median progression-free survival was 8.1 months $(95 \%$ confidence interval [CI] 6.5-12.0) in patients non-overexpressing p53 vs 5.8 months

(95\% CI 3.0-11.4) in patients overexpressing p53 (hazard ratio: $0.48 ; p=0.0399)$. b Median overall survival was 18 months $(95 \% \mathrm{CI}$ 13.6-28.0) in patients non-overexpressing p53 vs 11.4 months $(95 \%$ CI 8.2-18.0) in patients overexpressing p53 (hazard ratio: $0.26 ; p=$ $0.0027)$ 

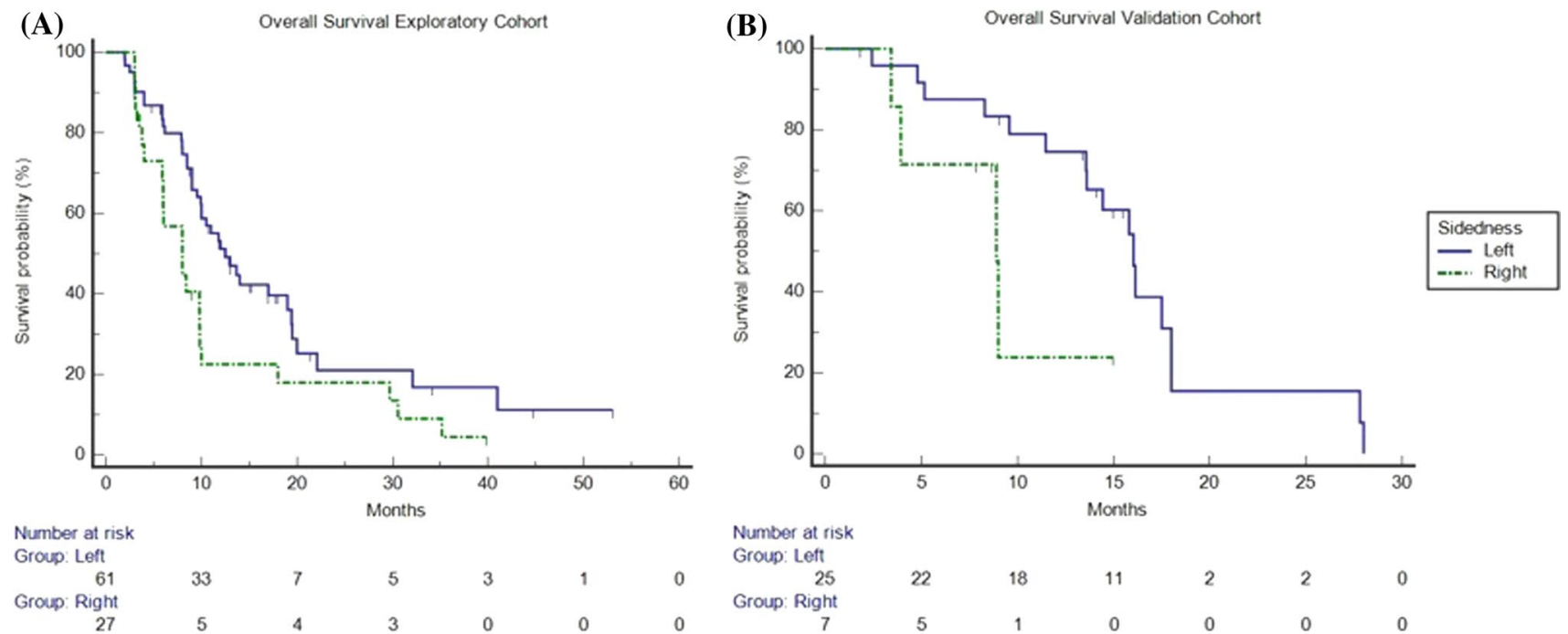

Number at risk Group: Left

$\begin{array}{rrrrrrr}25 & 22 & 18 & 11 & 2 & 2 & 0 \\ \text { Group: Right } & & & & \end{array}$

$\begin{array}{llllll}5 & 4 & 3 & 0 & 0 & 0\end{array}$

Fig. 4 Kaplan-Meier overall survival in patients with right-sided colorectal cancer [CRC] (dotted line) and in patients with left-sided CRC (continuous line) in the exploratory and validation cohorts. a In the exploratory cohort, median overall survival was 12.5 months (95\% confidence interval [CI] 9.9-19.0) vs 8 months (95\% CI 5.99.8) in patients with left-sided CRC and in patients with right-sided CRC, respectively (hazard ratio: $0.5 ; p=0.0232$ ). $\mathbf{b}$ In the validation

cohort, median overall survival in patients with right-sided CRC (dotted/green line) and in patients with left-sided CRC (continuous/blue line) is shown. Median overall survival was 16.0 months (95\% CI 13.5-28.0) vs 8.9 months (95\% CI 3.4-9.0) in patients with left-sided $\mathrm{CRC}$ and in patients with right-sided $\mathrm{CRC}$, respectively (hazard ratio: $0.37 ; p=0.0670$ )
(A)

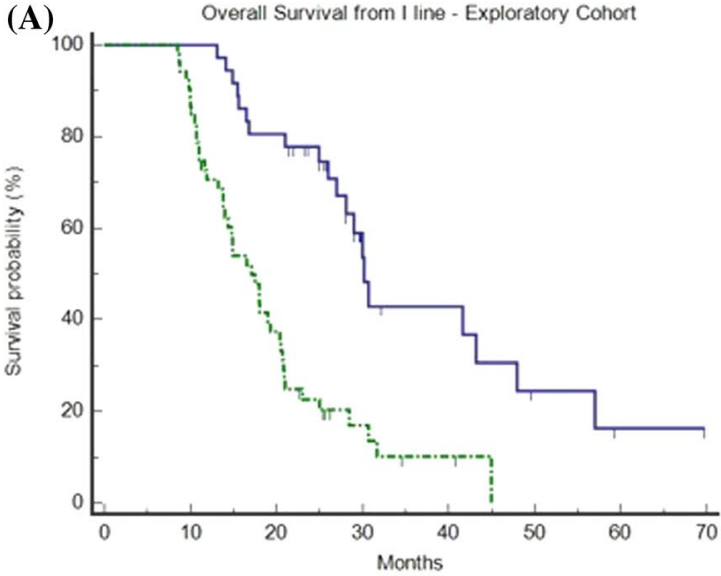

Number at risk

$\begin{array}{cccccccc}\begin{array}{c}\text { Group: Low } \\ 36\end{array} & 36 & 29 & 10 & 7 & 3 & 1 & 0 \\ \begin{array}{c}\text { Group: Abnormal } \\ 52\end{array} & 44 & 18 & 5 & 2 & 0 & 0 & 0\end{array}$

Fig. 5 Kaplan-Meier overall survival from first-line palliative therapy in patients overexpressing p53 (dotted line) and in patients nonoverexpressing p53 (continuous line) in the explorative (a) and the validation cohort (b). a Median overall survival was 30.2 months (95\% confidence interval [CI] 26.9-47.9) vs 17.1 (95\% CI 13.8-44.9) in patients with low p53 expression and in patients overexpressing

However, the clinical role of TP53 mutational status in CRC is still controversial. Several studies showed that patients with a tumour harbouring a TP53 mutation have a significantly poorer outcome than patients with WT TP53
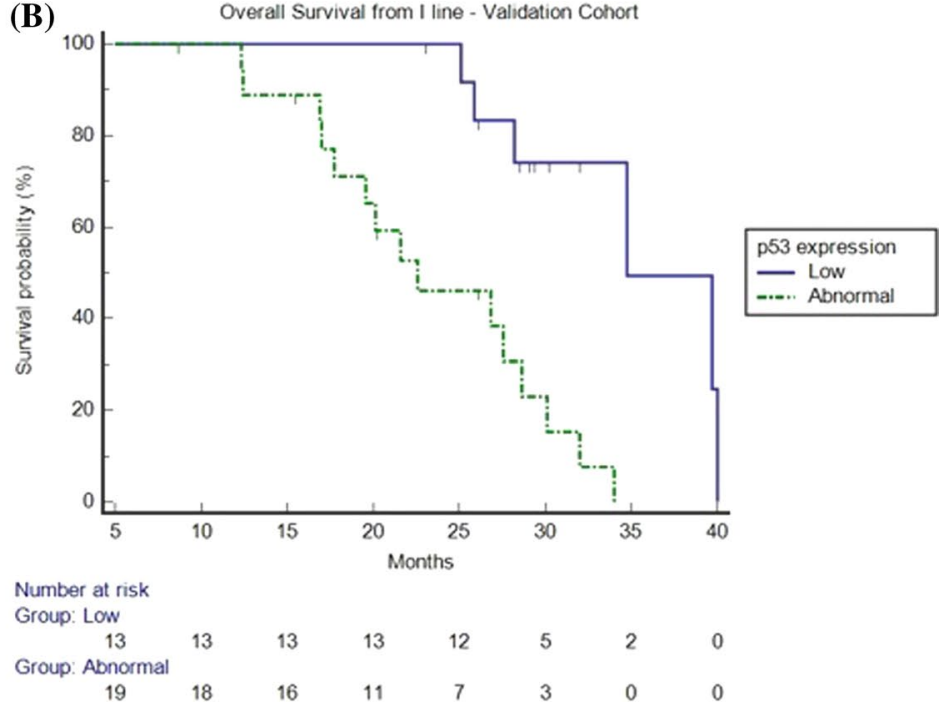

p53, respectively (hazard ratio: $0.28 ; p<0.0001$ ) in the exploratory cohort. b Median overall survival was 34.7 months (95\% CI 25.8-40) vs 22.5 (95\% CI 17-34) in patients with low p53 expression and in patients overexpressing $\mathrm{p} 53$, respectively (hazard ratio: $0.17 ; p=$ 0.0003 ) in the validation cohort

tumours. Moreover, p53 overexpression has a negative prognostic impact. Conversely, other findings failed to demonstrate a correlation between 553 overexpression and clinical outcome in patients with CRC [81-83]. Theodoropoulos 
et al. retrospectively evaluated the relationship between $\mathrm{p} 53$ and EGFR expression assessing the correlation with clinical/ histological prognostic factors and the impact on prognosis or survival in 164 patients with CRC with at least a 5-year follow-up. Overexpression of EGFR and p53 were significantly associated with an advanced $\mathrm{T}$ stage, suggesting that both proteins cause a growth advantage in deep invasion and they are a late event in CRC carcinogenesis [85].

In contrast with previous findings, Shyhmin et al. revealed that WT TP53 may enhance sensitivity to EGFR inhibitors and radiation through cell-cycle arrest, apoptosis and DNA damage repair, in CRC and non-small cell lung cancer [86]. The other two studies showed that TP53 mutations may be predictive of cetuximab sensitivity and TP53 genotyping could be useful to optimise the selection of patients with mCRC who should benefit from cetuximab-based chemotherapy $[87,88]$.

Of note, most pathologists do not have access to TP53 sequencing, and therefore, they use p53 immunohistochemistry as a surrogate for TP53 mutational analysis. Immunohistochemistry is quick, easy to perform and less expensive than sequencing. Hence, p53 immunohistochemistry represents a feasible biomarker already in use in the clinical setting. On this basis, our study aimed to evaluate whether the p53 abnormal expression could influence the clinical outcome of a patient with $R A S / B R A F$ WT mCRC, treated with anti-EGFR.

Globally, our analysis suggests that p53 overexpression may predict resistance to anti-EGFR treatment in patients with mCRC. In the exploratory cohort, a significant benefit in terms of all clinical outcomes (OS, PFS and RR) was observed in p53 WT patients, compared with those showing p53 over-expression. Furthermore, the p53 low expression status was more frequent in left-sided tumours. These results were confirmed in the validation cohort.

Furthermore, we analysed survival data from the first palliative therapy, which did not contain anti-EGFR antibodies. This analysis showed a significant benefit in terms of OS in p53 low expression patients compared with those showing p53 over-expression, in both cohorts. Conversely, no statistically difference in terms of PFS was observed in patients non-overexpressing p53 vs patients overexpressing p53 in the exploratory and validation cohorts. These data confirmed the prognostic role of p53 expression and suggested the absence of a predictive impact to response to therapies that do not contain anti-EGFR.

In the era of precision medicine, p53 analysis could improve the selection of patients who could benefit from anti-EGFR therapy. The identification of the optimal treatment strategy is successfully provided by a better knowledge of the mCRC heterogeneous nature. Therefore, the finding of p53 overexpression as a resistance mechanism to anti-EGFR therapies represents a further step toward personalised treatment.

The limitations of our study are mainly related to its retrospective nature. In particular, this latter feature and the absence of a control arm without EGFR therapy do not allow us to clearly demonstrate that p53 overexpression is associated with anti-EGFR resistance. Still, our analysis suggests a possible predictive p53 role in this setting. In future studies, it would also be interesting to investigate the association between the TP53 mutation, p53 IHC expression and clinical outcomes to confirm the use of p53 IHC, which is quick, easy to perform and inexpensive, as a surrogate for TP53 mutational analysis.

Although retrospective, we believe that our analysis could provide a relevant addition to the biological picture underlying the mechanism of resistance to anti-EGFR monoclonal antibodies in patients with mCRC. Further prospective studies, with a control arm without anti-EGFR therapy, will be needed to validate the prognostic and predictive effect of p53 expression in this setting, to better refine the molecular profile of patients more likely to benefit from this crucial treatment strategy.

Acknowledgements We thank all the investigators and patients for participating in this study.

Funding Open access funding provided by Università degli Studi di Cagliari within the CRUI-CARE Agreement.

\section{Declarations}

Funding No external funding was used for the conduct of this study or the preparation of this article.

Conflict of interest Pina Ziranu, Eleonora Lai, Marta Schirripa, Marco Puzzoni, Mara Persano, Andrea Pretta, Giada Munari, Nicole Liscia, Valeria Pusceddu, Fotios Loupakis, Laura Demurtas, Michela Libertini, Stefano Mariani, Marco Migliari, Marco Dubois, Riccardo Giampieri, Giovanni Sotgiu, Angelo Paolo Dei Tos, Sara Lonardi, Alberto Zaniboni, Matteo Fassan and Mario Scartozzi have no conflicts of interest that are directly relevant to the content of this article.

Ethics approval This study was performed in accordance with the study protocol, the ethical principles stated in the Declaration of Helsinki as well as those indicated in the International Conference on Harmonization (ICH) Note for Guidance on Good Clinical Practice (GCP; ICH E6, 1995), and all applicable regulatory requirements.

Consent to participate All patients signed a written informed consent before study entry. Adequate information was given to eligible patients by the principal investigator or co-investigators at each participating centre and in accordance with local regulations. The declaration of informed consent was personally signed and dated by the subject, and by the investigator/person designated by the investigator to conduct the informed consent discussion.

Consent for publication Patients signed an informed consent regarding the publication of their data. 
Availability of data and material The datasets generated during and/or analysed during the current study are available from the corresponding author on reasonable request.

Code availability Not applicable.

Author contributions Conceptualisation: AZ, PZ, EL, MP and MS; data curation: PZ, EL, MS, MP, MP, AP, GM, NL, VP, FL, LD, ML, SM, MM, MD, RG, GS, APDT, SL, AZ, MF and MS; formal analysis: PZ, EL, MP, RG, GS and MS; investigation: PZ, EL, MS, MP, MP, AP, GM, NL, VP, FL, LD, ML, SM, MM, MD, RG, GS, APDT, SL, AZ, MF and MS; methodology: PZ, EL, MP, GS, APDT, MF and MS; project administration: PZ, EL, MP and MS; supervision: PZ, EL, MS, MP, FL, APDT, SL, AZ, MF and MS validation: PZ, EL, MP and MS; writing - original draft: PZ, EL, MP and MS; writing-review and editing, PZ, EL, MS, MP, MP, AP, GM, NL, VP, FL, LD, ML, SM, MM, MD, RG, GS, APDT, SL, AZ, MF and MS.

Open Access This article is licensed under a Creative Commons Attribution-NonCommercial 4.0 International License, which permits any non-commercial use, sharing, adaptation, distribution and reproduction in any medium or format, as long as you give appropriate credit to the original author(s) and the source, provide a link to the Creative Commons licence, and indicate if changes were made. The images or other third party material in this article are included in the article's Creative Commons licence, unless indicated otherwise in a credit line to the material. If material is not included in the article's Creative Commons licence and your intended use is not permitted by statutory regulation or exceeds the permitted use, you will need to obtain permission directly from the copyright holder. To view a copy of this licence, visit http://creativecommons.org/licenses/by-nc/4.0/.

\section{References}

1. Ceresa BP, Vanlandingham PA. Molecular mechanisms that regulate epidermal growth factor receptor inactivation. Clin Med Oncol. 2008;2:47-61.

2. Carpenter G. ErbB-4: mechanism of action and biology. Exp Cell Res. 2003;284:66-77.

3. Citri A, Skaria KB, Yarden Y. The deaf and dumb: the biology of ErbB-2 and ErbB-3. Exp Cell Res. 2003;284:54-65.

4. Yarden Y. The EGFR family and its ligands in human cancer: signalling mechanisms and therapeutic opportunities. Eur J Cancer. 2001;37(Suppl. 4):S3-8.

5. Sebastian S, Settleman J, Reshkin SJ, Azzariti A, Bellizzi A, Paradiso A. The complexity of targeting EGFR signalling in cancer: from expression to turnover. Biochim Biophys Acta. 2006;1766:120-39.

6. Scartozzi M, Pierantoni C, Berardi R, Antognoli S, Bearzi I, Cascinu S. Epidermal growth factor receptor: a promising therapeutic target for colorectal cancer. Anal Quant Cytol Histol. 2006;28:61-8.

7. Rosell R, Carcereny E, Gervais R, Vergnenegre A, Massuti B, Felip E, et al. Erlotinib versus standard chemotherapy as first-line treatment for European patients with advanced EGFR mutation-positive non-small-cell lung cancer (EURTAC): a multicentre, open-label, randomized phase 3 trial. Lancet Oncol. 2012;13:239-46.

8. Bonner JA, Harari PM, Giralt J, Azarnia N, Shin DM, Cohen RB, et al. Radiotherapy plus cetuximab for squamous-cell carcinoma of the head and neck. N Engl J Med. 2006;354:567-78.
9. Ang KK, Zhang Q, Rosenthal DI, Nguyen-Tan PF, Sherman EJ, Weber RS, et al. Randomized phase III trial of concurrent accelerated radiation plus cisplatin with or without cetuximab for stage III to IV head and neck carcinoma: RTOG 0522. J Clin Oncol. 2014;32:2940-50.

10. Berardi R, Santinelli A, Brunelli A, Onofri A, Pierantoni C, Scartozzi M, et al. Epidermal growth factor receptor status in stages of resected non-small cell lung cancer: implications for treatment with epidermal growth factor receptor inhibitors. Anal Quant Cytol Histol. 2011;33:196-204.

11. Scartozzi M, Pierantoni C, Berardi R, Squadroni M, Cascinu S. Anti-EGFR strategies as an incremental step for the treatment of colorectal cancer patients: moving from scientific evidence to clinical practice. Expert Opin Ther Targets. 2006;10:281-7.

12. Silvestris N, Vincenzi B, Brunetti AE, Loupakis F, Dell'Aquila E, Russo A, et al. Pharmacogenomics of cetuximab in metastatic colorectal carcinoma. Pharmacogenomics. 2014;15:1701-15.

13. Scartozzi M, Bearzi I, Mandolesi A, Giampieri R, Faloppi L, Galizia E, et al. Epidermal growth factor receptor (EGFR) gene promoter methylation and cetuximab treatment in colorectal cancer patients. Br J Cancer. 2011;104:1786-90.

14. Scartozzi M, Bearzi I, Berardi R, Mandolesi A, Pierantoni C, Cascinu S. Epidermal growth factor receptor (EGFR) downstream signalling pathway in primary colorectal tumors and related metastatic sites: optimising EGFR-targeted treatment options. Br J Cancer. 2007;97:92-7.

15. Salvatore L, Aprile G, Arnoldi E, Aschele C, Carnaghi C, Cosimelli M, et al. Management of metastatic colorectal cancer patients: guidelines of the Italian Medical Oncology Association (AIOM). ESMO Open. 2017;2(1):e000147.

16. Cremolini C, Schirripa M, Antoniotti C, Moretto R, Salvatore L, Masi G, et al. First-line chemotherapy for mCRC: a review and evidence-based algorithm. Nat Rev Clin Oncol. 2015;12:607-19.

17. Rihawi K, Giampieri R, Scartozzi M, Pusceddu V, Bonotto M, Fasola G, et al. Role and mechanisms of resistance of epidermal growth factor receptor antagonists in the treatment of colorectal cancer. Expert Opin Invest Drugs. 2015;24:1185-98.

18. Puzzoni M, Ziranu P, Demurtas L, Lai E, Mariani S, Liscia N, et al. Why precision medicine should be applied across the continuum of care for metastatic colorectal cancer patients. Future Oncol. 2020;16:4337-9.

19. Lai E, Liscia N, Donisi C, Mariani S, Tolu S, Pretta A, et al. Molecular-biology-driven treatment for metastatic colorectal cancer. Cancers. 2020;12:1214.

20. Giampieri R, Aprile G, Del Prete M, Faloppi L, Bianconi M, Bonotto M, et al. Beyond RAS: the role of epidermal growth factor receptor (EGFR) and its network in the prediction of clinical outcome during anti-EGFR treatment in colorectal cancer patients. Curr Drug Targets. 2014;15:1225-30.

21. Scartozzi M, Giampieri R, Maccaroni E, Mandolesi A, Giustini L, Silva R, et al. Analysis of HER-3, insulin growth factor-1, nuclear factor- $\mathrm{kB}$ and epidermal growth factor receptor gene copy number in the prediction of clinical outcome for K-RAS wild-type colorectal cancer patients receiving irinotecan-cetuximab. Ann Oncol. 2012;23:1706-12.

22. Zhao B, Wang L, Qiu H, Zhang M, Sun L, Peng P, et al. Mechanisms of resistance to anti-EGFR therapy in colorectal cancer. Oncotarget. 2017;8:3980-4000.

23. Lai E, Pretta A, Impera V, Mariani S, Giampieri R, Casula L, et al. BRAF-mutant colorectal cancer, a different breed evolving. Expert Rev Mol Diagn. 2018;18:499-512.

24. Al-Marrawi MY, Saroya BS, Brennan MC, Yang Z, Dykes TM, El-Deiry WS. Off-label use of cetuximab plus sorafenib and panitumumab plus regorafenib to personalize therapy for a patient with V600E BRAF-mutant metastatic colon cancer. Cancer Biol Ther. 2013;14:703-10. 
25. Di Nicolantonio F, Martini M, Molinari F, Sartore-Bianchi A, Arena S, Saletti P, et al. Wild-type BRAF is required for response to panitumumab or cetuximab in metastatic colorectal cancer. J Clin Oncol. 2008;26:5705-12.

26. Solit DB, Garraway LA, Pratilas CA, Sawai A, Getz G, Basso A, et al. BRAF mutation predicts sensitivity to MEK inhibition. Nature. 2006;439:358-62.

27. Zhang YJ, Tian XQ, Sun DF, Zhao SL, Xiong H, Fang JY. Combined inhibition of MEK and mTOR signalling inhibits initiation and progression of colorectal cancer. Cancer Invest. 2009;27:273-85.

28. Markman B, Atzori F, Perez-Garcia J, Tabernero J, Baselga J. Status of PI3K inhibition and biomarker development in cancer therapeutics. Ann Oncol. 2010;21:683-91.

29. Loupakis F, Pollina L, Stasi I, Ruzzo A, Scartozzi M, Santini D, et al. PTEN expression and KRAS mutations on primary tumors and metastases in the prediction of benefit from cetuximab plus irinotecan for patients with metastatic colorectal cancer. J Clin Oncol. 2009;27:2622-9.

30. Li Q, Zhang D, Chen X, He L, Li T, Xu X, et al. Nuclear PKM2 contributes to gefitinib resistance via upregulation of STAT3 activation in colorectal cancer. Sci Rep. 2015;5:16082.

31. Yar Saglam AS, Alp E, Elmazoglu Z, Menevse S. Treatment with cucurbitacin $\mathrm{B}$ alone and in combination with gefitinib induces cell cycle inhibition and apoptosis via EGFR and JAK/STAT pathway in human colorectal cancer cell lines. Hum Exp Toxicol. 2016;35:526-43.

32. Scartozzi M, Giampieri R, Maccaroni E, Mandolesi A, Biagetti S, Alfonsi S, et al. Phosphorylated AKT and MAPK expression in primary tumours and in corresponding metastases and clinical outcome in colorectal cancer patients receiving irinotecancetuximab. J Transl Med. 2012;10:71.

33. Bertotti A, Migliardi G, Galimi F, Sassi F, Torti D, Isella C, et al. A molecularly annotated platform of patient-derived xenografts ("xenopatients") identifies HER2 as an effective therapeutic target in cetuximab-resistant colorectal cancer. Cancer Discov. 2011;1:508-23.

34. Richman SD, Southward K, Chambers P, Cross D, Barrett J, Hemmings $\mathrm{G}$, et al. HER2 overexpression and amplification as a potential therapeutic target in colorectal cancer: analysis of 3256 patients enrolled in the QUASAR, FOCUS and PICCOLO colorectal cancer trials. J Pathol. 2016;238:562-70.

35. Sartore-Bianchi A, Amatu A, Porcu L, Ghezzi S, Lonardi S, Leone $\mathrm{F}$, et al. HER2 positivity predicts unresponsiveness to EGFRtargeted treatment in metastatic colorectal cancer. Oncologist. 2019;24:1395-402.

36. Sartore-Bianchi A, Trusolino L, Martino C, Bencardino K, Lonardi S, Bergamo F, et al. Dual-targeted therapy with trastuzumab and lapatinib in treatment-refractory, KRAS codon 12/13 wild-type, HER2-positive metastatic colorectal cancer (HERACLES): a proof-of-concept, multicentre, open-label, phase 2 trial. Lancet Oncol. 2016;17:738-46.

37. Scartozzi M, Mandolesi A, Giampieri R, Bittoni A, Pierantoni C, Zaniboni A, et al. The role of HER-3 expression in the prediction of clinical outcome for advanced colorectal cancer patients receiving irinotecan and cetuximab. Oncologist. 2011;16:53-60.

38. Sclafani F, Kim TY, Cunningham D, Kim TW, Tabernero J, Schmoll HJ, et al. A randomized phase II/III study of dalotuzumab in combination with cetuximab 3999 and irinotecan in chemorefractory, KRAS wild-type, metastatic colorectal cancer. J Natl Cancer Inst. 2015;107:djv258.

39. Scartozzi M, Mandolesi A, Giampieri R, Pierantoni C, Loupakis $\mathrm{F}$, Zaniboni A, et al. Insulin-like growth factor 1 expression correlates with clinical outcome in K-RAS wild type colorectal cancer patients treated with cetuximab and irinotecan. Int J Cancer. 2010;127:1941-7.
40. Gherardi E, Birchmeier W, Birchmeier C, Van de Woude G. Targeting MET in cancer: rationale and progress. Nat Rev Cancer. 2012;12:89-103.

41. Ciardiello F, Bianco R, Caputo R, Caputo R, Damiano V, Troiani T, et al. Antitumor activity of ZD6474, a vascular endothelial growth factor receptor tyrosine kinase inhibitor, in human cancer cells with acquired resistance to antiepidermal growth factor receptor therapy. Clin Cancer Res. 2004;10:784-93.

42. Scartozzi M, Bearzi I, Mandolesi A, Pierantoni C, Loupakis F, Zaniboni A, et al. Epidermal growth factor receptor (EGFR) gene copy number (GCN) correlates with clinical activity of irinotecan-cetuximab in K-RAS wild-type colorectal cancer: a fluorescence in situ (FISH) and chromogenic in situ hybridization (CISH) analysis. BMC Cancer. 2009;9:303.

43. Demurtas L, Puzzoni M, Giampieri R, Ziranu P, Pusceddu $\mathrm{V}$, Mandolesi A, et al. The role of primary tumour sidedness, EGFR gene copy number and EGFR promoter methylation in RAS/BRAF wild-type colorectal cancer patients receiving irinotecan/cetuximab. Br J Cancer. 2017;117:315-21.

44. Mounawar M, Mukeria A, Le Calvez F, Hung RJ, Renard H, Cortot A, et al. Patterns of EGFR, HER2, TP53, and KRAS mutations of p14arf expression in non-small cell lung cancers in relation to smoking history. Cancer Res. 2007;67:5667-72.

45. Levine AJ. p53, the cellular gatekeeper for growth and division. Cell. 1997;88:323-31.

46. D'Orazi G, Givol D. p53 reactivation: the link to zinc. Cell Cycle. 2012;11:2581-2.

47. Michael D, Oren M. The p53 and Mdm 2 families in cancer. Curr Opin Genet Dev. 2002;12:53-9.

48. Michael D, Oren M. The p53-Mdm2 module and the ubiquitin system. Semin Cancer Biol. 2003;13:49-58.

49. Ashcroft M, Taya Y, Vousden KH. Stress signals utilize multiple pathways to stabilize p53. Mol Cell Biol. 2000;20:3224-33.

50. Ko LJ, Prives C. p53: puzzle and paradigm. Genes Dev. 1996;10:1054-72.

51. Klein C, Vassilev LT. Targeting the p53-MDM2 interaction to treat cancer. Br J Cancer. 2004;91:1415-9.

52. Smith ND, Rubenstein JN, Eggener SE, Kozlowski JM. The p53 tumor suppressor gene and nuclear protein: basic science review and relevance in the management of bladder cancer. $\mathbf{J}$ Urol. 2003;169:1219-28.

53. Bargonetti J, Manfredi JJ. Multiple roles of the tumor suppressor p53. Curr Opin Oncol. 2002;14:86-91.

54. Chiang MF, Chou PY, Wang WJ, Sze CI, Chang NS. Tumor suppressor WWOX and p53 alterations and drug resistance in glioblastomas. Front Oncol. 2013;3:43.

55. Hientz K, Mohr A, Bhakta-Guha D, Efferth T. The role of p53 in cancer drug resistance and targeted chemotherapy. Oncotarget. 2017;8:8921-46.

56. Lane DP. Cancer: p53, guardian of the genome. Nature. 1992;358:15-6.

57. Wang Z, Sun Y. Targeting p53 for novel anticancer therapy. Transl Oncol. 2010;3:1-12.

58. Lane D, Levine A. p53 research: the past thirty years and the next thirty years. Cold Spring Harb Perspect Biol. 2010;2:a000893.

59. Efeyan A, Serrano M. p53: guardian of the genome and policeman of the oncogenes. Cell Cycle. 2007;6:1006-10.

60. Takayama T, Miyanishi K, Hayashi T, Sato Y, Niitsu Y. Colorectal cancer: genetics of development and metastasis. J Gastroenterol. 2006;41:185-92.

61. Khoury K, Domling A. P53 mdm2 inhibitors. Curr Pharm Des. 2012;18:4668-78

62. Wang S, Zhao Y, Bernard D, Aguilar A, Kumar S. Targeting the MDM2-p53 protein-protein interaction for new cancer therapeutics. Top Med Chem. 2012;8:57-80. 
63. Muller PA, Vousden KH. p53 mutations in cancer. Nat Cell Biol. 2013;15:2-8.

64. Liu DP, Song $\mathrm{H}, \mathrm{Xu} \mathrm{Y}$. A common gain of function of $\mathrm{p} 53$ cancer mutants in inducing genetic instability. Oncogene. 2010;29:949-56.

65. Xu Y. DNA damage: a trigger of innate immunity but a requirement for adaptive immune homeostasis. Nat Rev Immunol. 2006;6:261-70.

66. Sigal A, Rotter V. Oncogenic mutations of the p53 tumor suppressor: the demons of the guardian of the genome. Cancer Res. 2000;60:6788-93.

67. Fidler IJ. The pathogenesis of cancer metastasis: the "seed and soil" hypothesis revisited. Nat Rev Cancer. 2003;3:453-8.

68. Kressner U, Lindmark G, Gerdin B, Pahlman L, Glimelius B. Immunohistological p53 staining is of limited value in the staging and prognostic prediction of colorectal cancer. Anticancer Res. 1996;16:951-7.

69. Kressner U, Inganas M, Byding S, Bliksta I, Pahlman L, Glimelius $\mathrm{B}$, et al. Prognostic value of p53 genetic changes in colorectal cancer. J Clin Oncol. 1999;17:593-9.

70. Nehls O, Klump B, Holzmann K, Lammering G, Borchard F, Gruenagel $\mathrm{HH}$, et al. Influence of p53 status on prognosis in preoperatively irradiated rectal carcinoma. Cancer. 1999;85:2541-8.

71. Ahnen DJ, Feigl P, Quan G, Fenoglio-Preiser C, Lovato LC, Bunn PA Jr, et al. Ki-ras mutation and p53 overexpression predict the clinical behaviour of colorectal cancer: a Southwest Oncology Group study. Cancer Res. 1998;58:1149-58.

72. Ludes-Meyers JH, Subler MA, Shivakumar CV, Munoz RM, Jiang $\mathrm{P}$, Bigger JE, et al. Transcriptional activation of the human epidermal growth factor receptor promoter by human p53. Mol Cell Biol. 1996;16:6009-19.

73. Deb SP, Munoz RM, Brown DR, Subler MA, Deb S. Wild-type human $\mathrm{p} 53$ activates the human epidermal growth factor receptor promoter. Oncogene. 1994;9:1341-50.

74. Johnson AC, Ishi S, Jinno Y, Pastan I, Merlino GT. Epidermal growth factor receptor gene promoter. Deletion analysis and identification of nuclear protein binding sites. J Biol Chem. 1998;263:5693-9.

75. Kageyama R, Merlino GT, Pastan I. Epidermal growth factor receptor gene transcription. Requirement for Sp1 and an EGF receptorspecific factor. J Biol Chem. 1988;263:6329-36.

76. Kageyama R, Pastan I. Molecular cloning and characterization of a human DNA binding factor that represses transcription. Cell. 1989;59:815-22.
77. Bheda A, Creek KE, Pirisi L. Loss of p53 induces epidermal growth factor receptor promoter activity in normal human keratinocytes. Oncogene. 2008;27:4315-23.

78. Nasif WA, Mukhtar MH, El-Emshaty HM, Al-Bukhari TA. Evaluation of circulating TP53 antigen in the serum of gastric cancer patients: as a tumour marker. Biomark Genom Med. 2013;5:175-80.

79. Baas IO, Mulder JW, Offerhaus GJ, Vogelstein B, Hamilton SR. An evaluation of six antibodies for immunohistochemistry of mutant p53 gene product in archival colorectal neoplasms. J Pathol. 1994;172:5-12.

80. Rijken AM, Offerhaus GJ, Polak MM, Gouma DJ, Van Gulik TM. p53 expression as a prognostic determinant in resected distal bile duct carcinoma. Eur J Surg Onc. 1999;25:297-301.

81. Canale M, Petracci E, Delmonte A, Chiadini E, Dazzi C, Papi M, et al. Impact of TP53 mutations on outcome in EGFR-mutated patients treated with first-line tyrosine kinase inhibitors. Clin Cancer Res. 2017;23:2195-202.

82. Gupta S, Khan H, Kushwaha VS, Husain N, Negi M, Ghatak A, et al. Impact of EGFR and p53 expressions on survival and quality of life in locally advanced oral squamous cell carcinoma patients treated with chemoradiation. Cancer Biol Ther. 2015;16:1269-80.

83. Monteiro LS, Diniz-Freitas M, Garcia-Caballero T, Warnakulasuriya S, Forteza J, Fraga M. Combined cytoplasmic and membranous EGFR and p53 overexpression is a poor prognostic marker in early stage oral squamous cell carcinoma. J Oral Pathol Med. 2012;41:559-67.

84. Cioca A, Cimpean A, Ceausu R, Fit AM, Zaharie T, Al-Hajjar N, et al. Crosstalk between EGFR and p53 in hepatocellular carcinoma. Asian Pac J Cancer Prev. 2014;15:8069-73.

85. Theodoropoulos GE, Karafoka E, Papailiou JG, Stamopoulos P, Zambirinis CP, Bramis K, et al. P53 and EGFR expression in colorectal cancer: a reappraisal of 'old' tissue markers in patients with long follow-up. Anticancer Res. 2009;29:785-91.

86. Shyhmin H, Benavente S, Armstrong EA, Li C, Wheeler DL, Harari PM. p53 modulates acquired resistance to EGFR inhibitors and radiation. Cancer Res. 2011;71:7071-9.

87. Oden-Gangloff A, Di Fiore F, Bibeau F, Lamy A, Bougeard G, Charbonnier F, et al. TP53 mutations predict disease control in metastatic colorectal cancer treated with cetuximab-based chemotherapy. Br J Cancer. 2009;100:1330-5.

88. Xiao-Lan L, Jianbiao Z, Zhi-Rong C, Wee-Joo C. p53 mutations in colorectal cancer- molecular pathogenesis and pharmacological reactivation. World J Gastroenterol. 2015;21:84-93.

\section{Authors and Affiliations}

\section{Pina Ziranu ${ }^{1}$ - Eleonora Lai ${ }^{1}$ Marta Schirripa ${ }^{2} \cdot$ Marco Puzzoni $^{1} \cdot$ Mara Persano $^{1}$ - Andrea Pretta ${ }^{1,3,4}$. Giada Munari ${ }^{2}$. Nicole Liscia ${ }^{1,3}$. Valeria Pusceddu ${ }^{1}$. Fotios Loupakis ${ }^{2}$ - Laura Demurtas ${ }^{1} \cdot$ Michela Libertini $^{5}$. Stefano Mariani ${ }^{1}$. Marco Migliari ${ }^{1} \cdot$ Marco Dubois $^{1} \cdot$ Riccardo Giampieri $^{6}$. Giovanni Sotgiu ${ }^{7} \cdot$ Angelo Paolo Dei Tos $^{8} \cdot$ Sara Lonardi $^{2}$. Alberto Zaniboni ${ }^{5} \cdot$ Matteo Fassan $^{8} \cdot$ Mario Scartozzi $^{1}$}

1 Medical Oncology Unit, University Hospital and University of Cagliari, S.S. 554 Bivio per Sestu Km 4, 500, 09042 Monserrato, Cagliari, Italy

2 Medical Oncology Unit 1, Department of Oncology, Veneto Institute of Oncology IOV-IRCCS, Padua, Italy

3 Medical Oncology Unit, Sapienza University of Rome, Rome, Italy

4 Department of Medical Oncology, Institut Jules Bordet, Université Libre de Bruxelles (ULB), Brussels, Belgium
5 Medical Oncology Unit, Fondazione Poliambulanza, Brescia, Italy

6 Medical Oncology Unit, University Hospital and Università Politecnica delle Marche, Ancona, Italy

7 Clinical Epidemiology and Medical Statistics Unit, Department of Medical, Surgical and Experimental Sciences, University of Sassari, Sassari, Italy

8 Surgical Pathology Unit, Department of Medicine (DIMED), University of Padua, Padua, Italy 